\title{
Injeções epidurais de esteroides no tratamento da dor lombar
}

\author{
Andrei F. Joaquim ${ }^{1}$ \\ Spine Division of the Department of Neurological Surgery, University of Virginia, Charlottesville, USA
}

\section{RESUMO}

Contexto: Injeções de corticosteroides no espaço epidural (IEE) fazem parte do arsenal terapêutico no tratamento da dor lombossacral associada à radiculopatia, e sua utilização é crescente em todo o mundo. Objetivo: Revisão dos principais aspectos referentes ao tema, como indicações e contraindicações, eficácia, forma de uso (interlaminar, transforaminal ou sacral), drogas a serem utilizadas e complicações. Método: Pesquisa bibliográfica baseada em dados MedLine (National Library of Medicine), abrangendo o período de 1980 a 2008. As palavras-chave utilizadas foram epidural, steroid injection, lumbar or lumbosacral. Os artigos foram avaliados pelos resumos, selecionando-se também revisões bibliográficas e trabalhos pertinentes ao texto. Realizou-se, além disso, pesquisa manual de eventuais referências cruzadas relevantes de artigos selecionados não encontradas na base de dados e em livros-texto sobre o assunto. Conclusão: A injeção de esteroide no espaço epidural é uma alternativa terapêutica importante no tratamento da dor lombossacral associada à radiculopatia. Novos estudos bem desenhados com diagnósticos clínico e radiológico mais precisos, posologias e técnicas padronizadas podem auxiliar na seleção de subgrupos de pacientes que mais se beneficiarão com o procedimento.

\section{PALAVRAS-CHAVE}

Injeção epidural de corticosteroides. Lombalgia. Dor ciática.

\section{ABSTRACT}

Steroid epidural injections in the treatment of lumbosacral pain

Background: Steroid epidural injections (SEI) are used for the treatment of low back pain associated with radiculopathy and its utilization is increasing around the world. Objective: Review the main aspects of the SEI: indications and contra-indications, efficacy, approaches (interlaminar, transforaminal or sacral), drugs to be used, and complications. Method: Bibliographical research using the MedLine Database (National Library of Medicine), covering the period from 1980 to 2008. The key words used were: epidural, steroid injection, lumbar or lumbosacral. Articles were evaluated by summaries, selecting the relevant review articles and also manual search of cross references articles not found on the data basis and text books on the subject. Conclusion: The injection of steroid in epidural space is an important therapeutic alternative in the treatment of lumbosacral pain associated to radiculopathy. New studies well designed with more precise clinical and radiological diagnosis and standardized techniques may help in the selection of subgroups of patients that more will benefit from the procedure.

\section{KEY WORDS}

Steroid epidural injection. Low back pain. Sciatic pain.

\section{Introdução}

Cerca de $80 \%$ das pessoas sentem dor lombar em algum momento ao longo da vida, e $2 \%$ a $8 \%$ delas desenvolverão lombalgia crônica. As implicações econômicas e sociais desses números são enormes: em países desenvolvidos, mais de $50 \%$ das disfunções musculoesqueléticas que causam repercussões socioeconômicas, como absenteísmo, afastamento e aposentadoria, são causadas por dor lombar. ${ }^{18}$ Quase 1\% da população em idade de trabalhar está afastado, temporária ou permanentemente, das atividades por causa da lombalgia. Além disso, a dor lombar corresponde à segunda causa de falta ao trabalho, perdendo apenas para o resfriado comum. ${ }^{15}$

Os fatores de risco mais bem estabelecidos associados à dor lombar crônica são idade avançada, sexo feminino, baixo nível socioeconômico e educacional, elevado índice de massa corpórea, tabagismo, depres- 
são, atividades físicas repetitivas, variações anatômicas e insatisfação com o trabalho. ${ }^{19}$ A lombalgia crônica é quase sempre de origem osteomuscular, porém diagnósticos diferenciais devem ser sempre afastados com base em histórico e exames clínico e radiológico. ${ }^{10,11}$

Clinicamente, a dor lombar pode ser acompanhada de irradiação para as pernas, as nádegas e os quadris. Ela recebe o nome de dor ciática quando essa irradiação se dá para a porção posterior da panturrilha e vai até a sua extremidade, e é geralmente causada por hérnias discais. A dor radicular é caracterizada pelo comprometimento sensitivo, motor ou misto do território inervado por uma raiz, geralmente com distribuição dermatomal. ${ }^{3}$

Assim, as injeções de esteroides no espaço epidural (IEE) fazem parte do arsenal terapêutico no tratamento da dor lombar aguda ou crônica associada à radiculopatia. Consideram-se as injeções como o "mais agressivo" dos tratamentos conservadores, pois os procedimentos cirúrgicos, embora com eficácia demonstrada quando bem indicados e adequadamente realizados, têm seus riscos inerentes e maior custo e requerem hospitalização, mesmo que breve. Dessa forma, procedimentos menos invasivos ganham espaço. Porém, há certo desconhecimento quanto ao uso das injeções epidurais. Qual é a sua real eficácia? Quais são as indicações? Em que local serão infiltradas (interlaminar, transforaminal ou hiato sacral)? Qual a droga a se utilizar? Tendo em vista essas inúmeras questões, realizamos revisão bibliográfica sobre o assunto, objetivando elucidar alguns pontos importantes.

\section{Método}

Realizou-se pesquisa bibliográfica utilizando a base de dados MedLine (National Library of Medicine), abrangendo o período de 1980 a 2008. As palavraschave utilizadas foram epidural, steroid, injection, lumbar or lumbosacral. Os artigos foram avaliados pelos resumos, selecionando-se também revisões bibliográficas e trabalhos pertinentes ao texto. Realizou-se, além disso, pesquisa manual de eventuais referências cruzadas relevantes de artigos selecionados não encontradas na base de dados e em livros-texto sobre o assunto.

\section{Resultados}

\section{Histórico}

O primeiro relato de uso de injeção epidural data de 1901, com injeção de cocaína para tratamento de ciatalgia.$^{23}$ Robecchi ${ }^{17}$ foi o pioneiro na descrição do uso de esteroide no espaço epidural, em 1952. Desde essa época, o uso das IEE tem aumentado significativamente em diferentes centros em todo o mundo, principalmente nos Estados Unidos, por ser uma alternativa plausível no controle da dor e na melhora da qualidade funcional dos pacientes. ${ }^{9}$ A utilização de IEE apresenta algumas vantagens em relação à cirurgia: os pacientes são tratados em regime ambulatorial, com procedimentos sob anestesia local, sem grandes perdas de dias de trabalho, sem incisões cutâneas, sem alteração irreversível da anatomia e com baixas taxas de complicações e raras contraindicações, possibilitando seu uso em pacientes com comorbidades médicas.

\section{Fisiopatologia}

O objetivo do uso do corticoide, em resumo, é diminuir o componente inflamatório presente na raiz neural, secundário à irritação química ou mecânica oriunda do processo compressivo, com consequente diminuição da dor. ${ }^{20,21}$

\section{Indicações}

O principal uso de injeções de corticosteroides se dá no tratamento da dor lombar aguda ou crônica com radiculopatia, geralmente associada à hérnia discal, à estenose lombar e aos cistos facetários ou de raiz. Há relatos do uso de injeções no manejo de radiculopatias secundárias a fraturas compressivas resultantes de osteoporose. ${ }^{16}$ Sugere-se que a correlação clínica e radiológica tenha influência no resultado terapêutico. As contraindicações gerais ao procedimento são: infecção sistêmica ou no local da punção, discrasias sanguíneas, alergia aos componentes da injeção e compressão radicular ou medular aguda com franca indicação cirúrgica.

As IEE podem ser usadas como terapêutica inicial associada às medidas de reabilitação física, fisioterapia e analgesia oral ou como resgate terapêutico em pacientes com quadros de piora.

\section{Locais de aplicação}

Há, basicamente, três locais para a aplicação das injeções: no forame intervertebral, no espaço epidural interlaminar e no hiato sacral. Não se sabe qual é o melhor lugar para a aplicação, uma vez que cada um possui vantagens específicas. Alguns autores defendem a aplicação transforaminal como mais efetiva, por concentrar a medicação nas proximidades do processo inflamatório, requerendo menor volume e atingindo 
especificamente o alvo desejado. ${ }^{8}$ Porém, essa aplicação é de difícil realização em pacientes com fusão óssea e instrumentação. ${ }^{2-13} \mathrm{~A}$ injeção na região interlaminar, possivelmente, tem menor risco de lesão neural e requer menos tempo de radioscopia, com menor incidência de complicações, ${ }^{5}$ mas é de difícil aplicação em pacientes submetidos a procedimento cirúrgico prévio, especialmente no nível L4-5, além de estar associada a punções intravasculares acidentais. ${ }^{13} \mathrm{~A}$ injeção no hiato sacral, por sua vez, é considerada segura, com menor risco de punção dural ou lesão neural do que ambas as anteriores. Contudo, requer altas doses de corticosteroides para se atingir os níveis terapêuticos desejados, sendo menos específica para o ponto de bloqueio.

O uso de fluoroscopia auxilia na correta localização anatômica dos pontos de infiltração e diminui a aplicação inadvertida intravascular de corticoide, ${ }^{24}$ sendo altamente recomendado nas aplicações. ${ }^{13}$

\section{Eficácia}

A real eficácia dessas injeções é difícil de ser avaliada, muitas vezes com trabalhos sem definições claras do diagnóstico dos pacientes, medidas inadequadas para avaliação do seguimento clínico e desfecho, gruposcontrole ausentes, sem definição exata do medicamento e da técnica utilizados e com análise estatística de qualidade questionável. As populações estudadas, por vezes, são heterogêneas, justificando os muitos resultados diferentes encontrados. ${ }^{3}$ Apesar disso, inúmeras revisões e metanálises sugerem benefício em situações específicas.

As conclusões, quanto à eficácia das injeções na dor lombar com radiculopatia, de quatro grandes revisões sistemáticas encontradas na literatura estão dispostas a seguir. A classificação da efetividade do tratamento quanto ao nível de evidência científica varia conforme as revisões, que serão especificadas caso a caso.

Na investigação mais antiga, em 1995, Koes e cols. ${ }^{12}$ avaliaram 12 estudos randomizados, classificando-os conforme a qualidade deles. Relataram que o resultado dos trabalhos revisados tem relação com a metodologia usada, com falhas nos desenhos. Dos quatro melhores estudos, dois relataram benefício, enquanto os outros dois tiveram resultados desfavoráveis. Eles concluíram, então, que, se houver benefícios com o uso das IEE no tratamento da lombalgia, eles serão de curta duração e transitórios, na ordem de semanas.

Em trabalhos mais recentes, porém, as conclusões são favoráveis ao uso. Boswell e cols., ${ }^{4}$ em 2003, também em revisão sistemática de estudos randomizados ou não, concluíram que há fortes evidências da efetividade - Nível II (Quadro 1) - da injeção transforaminal e evidências moderadas - Nível III (Quadro 1) - da injeção caudal no manejo da dor lombar com radiculopatia. As evidências são inconclusivas quanto ao manejo da dor crônica sem radiculopatia e síndrome pós-laminectomia.

Quadro 1

Classificação do nível de evidência

Nível I - Conclusiva: múltiplos estudos científicos

de alta qualidade ou revisão de metanálise

Nível II - Forte: evidência obtida de pelo menos um trabalho randomizado, controlado, com pelo menos 60 pacientes no menor grupo, ou um trabalho randomizado complementado por trabalhos prospectivos e retrospectivos

Nível III - Moderada: evidência de trabalhos randomizados pequenos e bem desenhados ou trabalhos clínicos não randomizados, mas bem desenhados ou coorte, grupos simples, série de casos e casos-controles, ou pelo menos uma metanálise

Nível IV - Limitada: evidência de estudos não experimentais de mais de um centro ou grupo

Nível V - Indeterminada: opinião de experts, baseada em evidências clínicas, estudos descritivos ou comitês de experts

Armon e cols., ${ }^{3}$ em 2007, concluíram que as injeções epidurais podem melhorar em curto prazo (entre duas $\mathrm{e}$ seis semanas) a dor radicular lombossacral - Nível C, Classe I-III (Quadro 2). A magnitude dos efeitos é pequena e sua generalização é difícil, uma vez que as técnicas, as doses, as populações e a mensuração dos prognósticos são variáveis. As injeções não mudaram a evolução da doença quanto à necessidade de cirurgia e alívio por mais de três meses. O uso de rotina das injeções não foi recomendado - Nível B, Classe I-III (Quadro 2).

Quadro 2 American Academy of Neurology

1. Classificação do nível de evidências quanto à qualidade dos estudos

Classe I - Estudos prospectivos, randomizados e controlados em populações representativas. Requer os seguintes critérios: a) resultado primário definido; b) critérios de inclusão e exclusão bem definidos; c) número de pacientes suficiente para evitar erros de interpretação; d) avaliação estatística adequada para os grupos em estudo

Classe II - Estudos de coorte prospectivos em população representativa e com desfechos bem definidos que preencham os critérios de a-d da Classe I ou trabalho randomizado prospectivo que não preencha os critérios da Classe I

Classe III - Outros trabalhos clínicos controlados com população representativa em que o prognóstico é avaliado independentemente ou não é influenciado pelo observador

Classe IV - Evidência de estudos não controlados, série de casos, relato de casos ou opinião de experts

2. Classificação das recomendações para intervenção terapêutica

A - Definitivamente efetivo, inefetivo ou perigoso para dada condição em população definida (requer pelo menos dois estudos de Classe I)

B - Provavelmente efetivo, inefetivo ou perigoso para dada condição em população definida (requer pelo menos um estudo de Classe I ou dois de Classe II)

C - Possivelmente efetivo, inefetivo ou perigoso para dada condição em população bem definida (requer pelo menos um estudo Classe II ou dois estudos Classe III)

D - Informação inadequada ou conflitante com o conhecimento atual 
Abdi e cols., ${ }^{1}$ em 2007, em nova revisão sistemática, concluíram que há fortes evidências - Nível II (Quadro 3) - quanto à eficácia no alívio da dor em menos de seis semanas e moderada - Nível III (Quadro 3) - após esse mesmo período com o uso de IEE interlaminar na dor radicular lombar. Quanto à injeção transforaminal e a caudal, as evidências também são fortes - Nível II (Quadro 3) - em relação à efetividade em curto prazo e moderadas - Nível II (Quadro 3) - em longo prazo.

\begin{tabular}{l} 
Quadro 3 \\
\multicolumn{1}{c}{ Classificação do nível de evidência ${ }^{1}$} \\
\hline Nível I - Conclusiva: múltiplos estudos científicos \\
de alta qualidade ou revisão de metanálise \\
Nível II - Forte: evidência obtida de pelo menos um \\
trabalho randomizado, controlado, ou evidências de \\
trabalhos menores, porém com desenho adequado \\
Nível III - Moderada: \\
a) evidência obtida de trabalhos randomizados \\
bem desenhados pseudorrandomizados \\
b) evidência obtida de estudos comparativos controlados \\
e não randomizados (estudos de coorte, caso-controle \\
ou séries de caso com grupo-controle) \\
c) evidência obtida de estudos comparativos com controle \\
histórico, ou série de casos ininterruptos sem grupo-controle \\
Nível IV - Limitada: evidência de estudos bem definidos, \\
não experimentais, de mais de um centro de pesquisa \\
ou conflitantes evidências de múltiplos trials \\
Nível V - Indeterminada: opinião de autoridades respeitadas, baseadas \\
em evidências clínicas, estudos descritivos ou reunião de experts \\
\hline
\end{tabular}

\section{Medicamentos utilizados}

A composição das injeções epidurais em muitos serviços consiste em um esteroide, associado ou não a um anestésico local. Em procedimentos com fluoroscopia, um contraste não iônico é usado para se assegurar o correto local da injeção.

Os corticosteroides utilizados são a dexametasona, triamcinolona, betametasona e metil-prednisolona. A dexametasona tem partículas menores, com melhor solubilidade, que diminui o risco de infartos embólicos quando erroneamente injetada no espaço intravascular. ${ }^{7} \mathrm{~A}$ betametasona tem início rápido e efeito antiinflamatório estendido. A triancinolona tem um efeito anti-inflamatório sustentado. A dose de esteroides para injeções interlaminares pode ser de $40 \mathrm{~g}$ a $80 \mathrm{~g}$ de dexametasona, $12 \mathrm{mg}$ a $18 \mathrm{mg}$ de betametasona ou $80 \mathrm{mg}$ a $120 \mathrm{mg}$ de metilprednisolona, sendo cerca da metade para injeções foraminais, diluída em lidocaína a $1 \%$ ou $2 \%$ ou solução fisiológica. O volume a ser injetado é cerca de $1,5 \mathrm{ml}$ a $2 \mathrm{ml}$ em injeções transforaminais, $6 \mathrm{ml}$ a $10 \mathrm{ml}$ para injeções interlaminares e até $20 \mathrm{ml}$ para injeções caudais. ${ }^{22}$

\section{Repetição das injeções}

O intervalo de tempo ideal é desconhecido; ele varia entre os serviços e trabalhos, dependendo também da eficácia atingida com a primeira injeção e do corticosteroide utilizado. Por exemplo, a metilprednisolona tem sua ação por cerca de duas semanas, não se justificando sua repetição antes desse prazo. O número máximo de injeções também é controverso. ${ }^{14}$ Apesar disso, alguns trabalhos sugerem maior benefício com a repetição seriada das injeções. ${ }^{13,25}$ Muitos serviços repetem de três a quatro vezes, a critério clínico.

\section{Complicações}

A complicação mais comum consiste em cefaleia transitória $(0,5 \%$ a $1 \%$ para injeções interlaminares e $0,6 \%$ para injeções caudais). ${ }^{6}$ Complicações menores incluem sangramento no local da punção, reações vasovagais, reações alérgicas às medicações, eritema facial e dor local. Mais raramente, há relatos de casos de lesão na raiz neural, meningite asséptica, efeitos sistêmicos do corticosteroide, aracnoidite, síndrome da cauda equina, abscesso epidural e até de hematoma retroperitoneal (após injeção transforaminal). ${ }^{3}$ Cerca de $4 \%$ dos pacientes relatam piora dos sintomas nas primeiras 24 horas após a aplicação. ${ }^{3}$

\section{Conclusões}

A injeção de esteroide no espaço epidural é uma alternativa terapêutica importante no tratamento da dor lombossacral associada à radiculopatia. Seu emprego é bastante atraente, tendo em vista a efetividade, tanto em curto quanto em longo prazo. Cada forma de aplicação possui vantagens que devem ser consideradas caso a caso e conforme as necessidades do paciente e experiência do médico. Infelizmente, como quase todo tratamento, nem sempre resultados satisfatórios são obtidos. Novos estudos controlados, com diagnósticos clínicos e radiológicos mais precisos, posologias e técnicas padronizadas, podem auxiliar na seleção de subgrupos de pacientes que mais se beneficiarão do procedimento.

\section{Referências}

1. Abdi S, Datta S, Trescot AM, Schultz DM, Adlaka R, Atluri SL, et al. Epidural steroids in the management of chronic spinal pain: a systematic review. Pain Physician. 2007;10:185-212. 
2. Ackerman WE, Ahmad M. The efficacy of lumbar epidural steroid injections in patients with lumbar disc herniations. Anesth Analg. 2007;104:1212-22.

3. Armon C, Argoff CE, Samuels J, Backonja MM. Assessment: Use of epidural steroid injections to treat radicular lumbosacral pain. Report of the Therapeutics and Technology Assessment Subcommittee of the American Academy of Neurology. Neurology. 2007;68:723-9.

4. Boswell MV, Hansen HC, Trescot AM, Hirsch JA. Epidural steroids in the management of chronic spinal pain and radiculopathy. Pain Physician. 2003;6:319-34.

5. Candido KD, Raghavendra MS, Chinthagada M, Badiee S, Trepashko DW. A prospective evaluation of iodinated contrast flow patterns with fluoroscopically guided lumbar epidural steroid injections: the lateral parasagital interlaminar epidural approach versus the transforaminal epidural approach. Anesth Analg. 2008;106:638-44.

6. Carrete S, Leclaire R, Marcoux S, Morin F, Blaise GA, StPierre A, et al. Epidural injections for sciatica due to herniated nucleus pulposus. N Engl J Med. 1997;336:1634-40.

7. Derby $\mathrm{R}$, Lee $\mathrm{SH}$, Date ES, Lee JH, Lee $\mathrm{CH}$. Size and aggregation of corticosteroids used for epidural injections. Pain Med. 2008:9:227-34.

8. Dominguez E. The practice of cervical transforaminal injection of steroids deserves more scrutiny. Pain Physician. 2005;8:323-4.

9. Friedly J, Chan L, Deyo R. Increases in lumbosacral injections in the medicare population 1994-2001. Spine. 2007;32:1754-60.

10. Joaquim AF. Abordagem inicial do paciente com mielopatia aguda não compressiva. Rev Bras Med. 2007;64:164-9.

11. Joaquim AF, Maturana FAP, Anderle DV, Zambelli HJL, Maldaun MVC. Metástases na coluna vertebral. Rev Neurocienc. 2007;15:67-72.

12. Koes BW, Scholten RJ, Mens JM, Bouter LM. Efficacy of epidural steroid injections for low-back pain and sciatica: a systematic review of randomized clinical trials. Pain. 1995;63:279-88.

13. Manchikanti L, Singh V, Rivera JJ, Pampati V, Beyer C, Damron K. Effectiveness of caudal epidural injections in discogram positive and negative chronic low back pain. Pain Physician. 2002;5:18-29.

14. Novak S, Nemeth WC. The basis for recommending repeating epidural steroid injections for radicular low back pain: a literature review. Arch Phys Med Rehabil. 2008;89:543-52.
15. Papageorgiou AC, Croft PR, Ferry S, Jayson MIV, Silman A. Estimating the prevalence of low back pain in the general population. Spine. 1995;20:1889-94.

16. Prather H, Watson JO, Gilula LA. Nonoperative management of osteoporotic vertebral compression fractures. Injury. 2007;38(3):S40-8.

17. Robecchi A: L'ídrocortisone: prime esperienze cliniche in campo reumatologico [abstract]. Minerva Med. 1952;13:17

18. Roland M, Morris R. A study of natural history of low-back pain. Part I: Development of a reliable and sensitive measure of disability in low-back pain. Spine. 1983;8:141-4.

19. Rubin DI. Epidemiology and risk factors for spine pain. Neurol Clin. 2007;25:353-71.

20. Saal JS. The role of inflammation in lumbar pain. Spine. 1995;20:1821-7.

21. Saal JS, Franson RC, Dobrow R, Saal JA, White AH, Goldthwaite $\mathrm{N}$. High levels of inflammatory phospholipase A2 activity in lumbar disc herniations. Spine. 1990;15:674-8.

22. Schäufele MK. Interventional and semi-invasive procedures for low back pain and disc herniation. In: Mayer HC, editor. Minimally invasive spine surgery. 2nd ed. Berlin: SpringerVerlag Berlin Heidelberg; 2006, p. 249-59.

23. Sicard $A$. Les injections médicamenteuses extra-durales par voie sacro-coccygienne [abstract]. $\mathrm{Cr}$ Soc Biol Paris. 1991;53:369

24. Smuck M, Fuller BJ, Chiodo A, Benny B, Singaracharlu B, Tong $\mathrm{H}$, et al. Accuracy of intermittent fluoroscopy to detect intravascular injection during transforaminal epidural injections. Spine. 2008;33:205-10.

25. Waldman SD. The caudal epidural administration of steroids in combination with local anesthetics in the palliation of pain secondary to radiographically documented lumbar herniated disc: A prospective outcome study with 6-months follow-up. Pain Clinic. 1998;11:43-4.

Original recebido em julho de 2008

Aceito para publicação em dezembro de 2008

Endereço para correspondência

Andrei F. Joaquim

Rua Jasmin, 190, ap. 133A

13087-460 - Campinas, SP, Brasil

E-mail: andjoaquim@yahoo.com 